\title{
Familia abelera: una metáfora sobre la relación entre un artista argentino y su público*
}

\author{
Daniela Soledad Gonzalez** \\ Recibido: 2020-02 13 • Enviado a pares: 2020-03-19 \\ Aprobado por pares: 2020-04-27 • Aceptado: 2020-05-22 \\ https://doi.org/10.22395/angr.v19n37a12
}

\begin{abstract}
Resumen
En este artículo se analiza la metáfora "el público es la familia", elaborada por el cantante Abel Pintos (1984, Bahía Blanca, Argentina) para hacer referencia a la relación con sus seguidores. Primeramente, se hará un breve repaso por la vida del artista, su obra y su actividad actual de promoción artística. Luego, se revisarán las características principales de los movimientos de masas, en particular, en lo que hace a la relación de los artistas con su público (nivel de cohesión alto, organización para fines comunes, respaldo y admiración por un personaje público, referencias internas, símbolos en común y uso de productos con la marca del artista). A continuación, se detallará la metodología de la investigación y se presentarán los resultados del análisis discursivo de algunas publicaciones y comentarios de Facebook en los que la metáfora que focalizamos se hace presente. Se extraerán de estos las características de la relación entre Abel Pintos y sus seguidores, y se enumerarán otras conceptualizaciones existentes para hablar de dicha relación. La más importante de ellas consiste en constituir una comunidad con una unión tan fuerte como la de una familia. Otras características que se advertirán son las siguientes: iniciativas de organización para hacer productos u ofrecer servicios en conjunto, referencias internas, creación de términos identificatorios (por ejemplo, el adjetivo abelero/a), lenguaje performativo, gran admiración por el cantante argentino. Por otro lado, se señalarán ocasionales inconsistencias entre la metáfora "el público es la familia" y algunos eventos que contradicen dicha conceptualización. Finalmente, se presentarán las conclusiones del estudio.
\end{abstract}

Palabras clave: artista; cantante; audiencia; redes sociales; familia, discurso.

\footnotetext{
Este artículo forma parte de la investigación posdoctoral de su autora, financiada por una beca del Conicet, que versa sobre la metáfora y la metonimia conceptuales en relación con los diversos modos de construcción gramatical.

" Doctora en Letras, Universidad Nacional de Cuyo, Mendoza, Argentina. Profesora, Universidad Nacional de Cuyo, Mendoza, Argentina. Becaria de investigación posdoctoral del Conicet. Correo electrónico: gonzalezdanielasoledad@yahoo.com.ar. Orcid: http://orcid.org/0000-0003-2437-531X
} 


\title{
Abelian Family: a Metaphor About the Relationship Between an Argentinian Artist and Its Public
}

\begin{abstract}
This article analyzes the metaphor "the public is the family", composed by the singer Abel Pintos (1984, Bahía Blanca, Argentina) to communicate something about his relationship with his followers. Primarily, the study performs a brief account of the artist's life, its works and its current activity as an art promoter After that, the study reviews the main characteristics of mass movements, particularly those related with the relationship between artists and their public (high level of cohesion, organized around common goals, support and admiration for a public character, internal references, common symbols, and use of products with the artist brand. After that, this text exposes its research methodology and presents the results of the discursive analysis of some of the publications and comments on Facebook in which the metaphor focalized earlier is present. From these, the study extracts the characteristics of the relationship between Abe Pintos and its followers and other conceptualizations will be set on stage to talk about this relationship The most relevant of them consists of constituting a community with a bond as strong as a family bond Another of the characteristics is the organization initiatives for making products or offering communal services, internal references, creation of identity vocabulary, (e. g., the adjective abelian), performative language, and great admiration for the Argentinian singer. Besides, the study points out the occasional inconsistencies between the metaphor "the public is the family and some events that contradict that conceptualization. At last, the study presents its conclusions.
\end{abstract}

Keywords: artist; singer; audience; social networks; family, discourse.

\section{Família abelera: uma metáfora sobre a relação entre um artista argentino e seu público}

\section{Resumo}

Neste artigo, é analisada a metáfora "o público é a família", elaborada pelo cantor Abel Pintos (1984, Bahía Blanca, Argentina) para fazer referência à relação com seus fãs. Em primeiro lugar, é feita uma breve revisão da vida do artista, sua obra e sua atividade atual de promoção artística. Logo, são revisadas as características principais dos movimentos de massa, em particular, no que se refere à relação dos artistas com seu público (nível de coesão alto, organização para fins comuns, apoio e admiração por uma personagem pública, referências internas, símbolos em comum e uso de produtos com a marca do artista). A seguir, é detalhada a metodologia da pesquisa e são apresentados os resultados da análise discursiva de algumas publicações e comentários do Facebook nos quais a metáfora que focamos está presente. Disso são extraídas as características da relação entre Abel Pintos e seus fãs, e são enumeradas outras conceituações para falar dessa relação. A mais importante delas consiste em constituir uma comunidade com uma união tão forte como a de uma família. Outras características são: iniciativas de organização para fazer produtos ou oferecer serviços em conjunto, referências internas, criação de expressões de identidade (por exemplo, abelero/a), linguagem performática, grande admiração pelo cantor argentino. Por sua vez, são indicadas inconsistências ocasionais entre a metáfora "o público é a família" e alguns eventos que contradizem essa conceituação. Por último, são apresentadas as conclusões do estudo.

Palavras-chave: artista; cantor; audiência; redes sociais; família, discurso. 


\section{Introducción}

El propósito de esta investigación es analizar una metáfora elaborada por el cantante Abel Pintos (1984, Bahía Blanca, Argentina) para hacer referencia a la relación con su público. Se trata de la metáfora "el público es la familia". El enfoque teórico de este trabajo es la Lingüística Cognitiva'. Esta perspectiva de estudio sostiene que toda emisión implica una conceptualización de la experiencia que se busca comunicar, desde la elección de las desinencias flexivas hasta la estructura de la oración. En particular, se aplica la teoría de la metáfora conceptual (Lakoff y Johnson, 2001).

Este artículo se estructura de la siguiente manera: primero, se resume la vida del artista y su obra. En segundo lugar, se revisarán las características principales de los movimientos de masas con el foco en la relación de los artistas con su público. En tercer lugar, se detalla la metodología de la investigación y se presenta el resultado del análisis de algunos discursos extraídos de las redes, en los que la metáfora mencionada se hace presente. Se enumeran las características de la relación entre Abel Pintos y sus seguidores junto a otras conceptualizaciones que pueden existir sobre dicha relación. Por último, se presentan las conclusiones del estudio.

\section{Abel Pintos}

Abel Federico Pintos (1984, Bahía Blanca) es un cantante y compositor argentino de folklore y pop latino. Su carrera musical comenzó siendo un niño. En 1997 presentó su primer álbum, Para cantar he nacido, y actuó en el escenario mayor de Cosquín. Desde entonces, no ha dejado de producir discos con un éxito cada vez mayor. Entre ellos se encuentran Revolución (2010), Sueño dorado (2012), Abel (2013) y 11 (2016). Hace varios años comenzó a interpretar canciones de su propia autoría. Ha ganado varios premios y ha realizado colaboraciones musicales con numerosos artistas internacionales.

Como artista internacional y como uno de los más importantes en Argentina, Abel Pintos permanece siempre muy activo en sus redes sociales, proceder casi indispensable hoy en día para quienes forman parte del mundo del espectáculo. En relación con esto, afirma Gallardo Camacho (2013):

Algunos presentadores de televisión son conscientes de que su imagen es más que lo que se emite desde un estudio. Por ese motivo están presentes en las redes sociales y crean una extensión virtual de su imagen para estar más cerca de los espectadores. Se genera por tanto una interacción real y, en muchos casos, en directo. (p. 4)

Obsérvese que se habla de Lingüística Cognitiva, con mayúsculas. Esta distinción responde a la realizada por Geeraerts (2006, p. 3). El uso de las mayúsculas señala un enfoque de estudio de la lengua que incluye varias teorías lingüísticas como la de los prototipos y la de los marcos semánticos, reunidas bajo una perspectiva común que nació en la segunda mitad del siglo XX. En el segundo caso se hace una alusión más genérica a cualquier estudio de la lengua como un fenómeno mental. 
Las principales redes sociales de carácter público de las que participa el bahiense son Twitter, Facebook e Instagram. En Facebook, su usuario oficial es@oficial.abelpintos (https://www.facebook.com/oficial.abelpintos).En Instagram, su usuario es@ abelpintos (https://www.instagram.com/abelpintos/?hl=es-la) y en Twitter, @AbelPintos (https://twitter.com/AbelPintos?s=20). Su página web oficial es https://www.abelpintos.com/. Por otra parte, la música de Pintos está disponible en diversas plataformas digitales como Spotify, Keek y YouTube.

La música de Abel Pintos nace, principalmente, de necesidades emocionales, como el mismo artista ha afirmado en numerosas entrevistas, en las que ha señalado que la música le ha permitido canalizar lo que no puede decir con palabras. El repertorio del cantante es predominantemente sentimental e intimista; sin embargo, tiene algunas canciones de tono más social. Si bien esta faceta social de la música del bahiense no es la que predomina en su música hasta el momento, en los recitales toma más prominencia e incluso se resignifican expresiones que originalmente se referirían a la figura de la amante para hacer referencia al público en su conjunto. Por ejemplo, la canción Adiós del cantante narra un discurso dirigido a la persona amada despidiéndose de ella y deseándole lo mejor; en los recitales es la canción de cierre y es utilizada como una despedida del público.

Abel Pintos ha aseverado en muchas ocasiones que la relación que tiene con su público es la de una familia. De hecho, su último disco, grabado en vivo en sus recitales en el Estadio River Plate en el año 2018, se denomina La familia festeja fuerte. Pintos explica así la elección de la metáfora de la familia en una entrevista que hace Ajmat (2017) para La Nación:

Nunca comprendí el término fan. No me identifica. Al público que me acompaña lo fui llamando de distintas maneras. En 2010, compuse una canción que se llama "peregrinos" y en esa época la gente empezó a viajar mucho para verme. Entonces los empecé a llamar peregrinos. Luego vino el tema de la complicidad. El público iba al show. Sin saber de qué se trataba, iban. Entonces empecé a sentirlo así, son cómplices: "no tenés que explicar nada. Nos gustará más, nos gustará menos, pero vamos". Me sentí muy privilegiado de poder contar con semejante voto de confianza y con esa libertad para crear.

En esta explicación, el cantautor recorre otras metáforas que ha ido utilizando a lo largo del tiempo para referirse a la comunidad que lo sigue ("el público son peregrinos, el público son cómplices"), pero que no llenaban sus expectativas completamente. Al momento, la metáfora de la familia abelera ha resultado ser la mejor para él. El interés que el autor ha puesto en definir el estatus de su público habla de la importancia que tienen para él sus seguidores y la relación que con ellos ha entablado. 
Sobre la importancia que la nueva denominación de la relación con su público conlleva para su acuñador, se pueden citar numerosos discursos (entrevistas al autor, noticias, publicaciones en redes del autor, etc.). Aquí solo se presentará un fragmento de una noticia que se publicó en Clarín de Gómez (2018), que reúne varios aspectos de los señalados arriba:

A pesar de la vara alta que dejó el show de River en 2017, su "familia" logró dejarlo sin palabras otra vez. Con las manos en la cabeza y una sonrisa imborrable, se tomó varios momentos de pausa para observarlos, tratando de entender qué había hecho tan bien para tener semejante recompensa. Finalmente, sus lágrimas fueron inevitables, y después de hacer una reverencia a su público, expresó "iAguante la familia carajo!" [...]. Lo que digo en Motivos, ustedes me lo hacen sentir. Ustedes me acompañan sin importar qué ofrecemos y qué compartimos. Los quiero mucho, posta", concluyó Abel.

La noticia comenta un recital de Abel Pintos que se realizó en el Estadio Obras en octubre de 2018. En el fragmento seleccionado de la noticia, se observa la recurrencia del término familia (en boca de la autora de la noticia, Macarena Gómez Miñano y en boca de Abel Pintos). Además, se aprecia la emoción que le provoca al artista el cariño y el acompañamiento de su público y la declaración de sentimientos hacia él, que brota del cantautor: "Lo que digo en Motivos, ustedes me lo hacen sentir. Ustedes me acompañan sin importar qué ofrecemos y qué compartimos. Los quiero mucho, posta" [cursivas propias].

\section{Los artistas y su público}

La relación entre los artistas y su público es un tipo especial de conexión humana. En esta sección se señalan las peculiaridades de esta relación individuo-público-masa que emergen en el análisis del discurso que se presenta en el apartado siguiente. No se problematiza aquí la noción de masa, que puede resultar muy ambivalente, sobre todo en lo que se refiere a su valoración como concepto positivo o negativo. Aquí no interesa valorar la masa, sino advertir patrones de funcionamiento de las masas que siguen a famosos y atisbar algo del comportamiento de los famosos en relación con ellas. Para dar un sentido básico y operativo al término masa, se parte de las palabras de McQuail (2000):

La palabra 'masa' es casi imposible de ser definida a causa de sus diferentes connotaciones, su rasgo esencial es la ambivalencia. En el pensamiento social ha tenido y tiene significados tanto negativos como positivos. Sus significados negativos derivan históricamente de su uso en referencia a la multitud o el "populacho" en tanto masa de gente revoltosa e ignorante, inteligencia o racionalidad (Branison 1961). En su 
sentido positivo, especialmente en la tradición socialista, connota la fuerza y solidaridad de los trabajadores cuando se organizan para fines comunes. En contextos donde la cantidad es entendida como positiva es una palabra de aprobación, sea en 'respaldo masivo', 'movimiento masivo', 'acción masiva', etc. (p. 75)

A partir de este fragmento, la caracterización que se hace del conjunto de los seguidores de Abel Pintos es la de un grupo de personas con un nivel de cohesión medio que aumenta en los pequeños grupos que lo conforman y se organizan para fines comunes (principalmente relacionados con la figura que los une). La característica común a todos los integrantes de la masa abelera es su respaldo y admiración por el cantautor bahiense, que funciona como señal identificadora de estos como miembros de esta comunidad ante los demás.

En general, las características que se aprecian en la interacción de los grupos que siguen fervientemente a un artista como Abel Pintos son las siguientes:

(a) Autorreferencias o referencias internas del grupo: se hace alusión a momentos y emociones utilizando frases de las canciones del artista al que se sigue.

(b) Productos con la marca del artista en cuestión: remeras con letras de sus canciones y fotos del artista, tatuajes, gorros, vinchas, etc.

(c) Símbolos que representan al autor. En el caso de Abel Pintos, los símbolos son, por ejemplo, el sombrero típico que utiliza en sus conciertos, el pájaro cantor (en referencia a una de sus canciones que se titula así y trata sobre el cantante mismo), la leyenda gratitud que Pintos se tatuó en el cuello.

(d) Grupos que realizan reuniones físicas de los seguidores del artista con diversos niveles de institucionalización. Con este término se indica que estos grupos cuentan con nombre registrado y vestimenta especial, hacen viajes en conjunto para ver los espectáculos y llevan banderas con eslóganes propios. En el caso de Abel Pintos, por ejemplo, existen los grupos Mariposas de Abel Mendoza (https://www.facebook. com/groups/768582493312856/), Gratitud abelera (https://www.facebook.com/GratitudAbelera-131387667511378/) y Alma abelera (https://www.facebook.com/alma.abelera.7). Este último grupo realizó recientemente un sorteo para recolectar alimentos, que luego donó a un comedor comunitario.

\section{Metodología}

La metodología de esta investigación es cualitativa. Se utiliza un método hermenéutico para comprender el sentido de diversos discursos extraídos de redes sociales (lectura comprensiva de los textos, interpretación contextualizada y análisis crítico). En tér- 
minos de la lingüística cognitiva, se llevará a cabo un análisis del discurso orientado a identificar conceptualizaciones acerca de la relación del artista con su público. Se considera aquí que el análisis hermenéutico de los textos y el análisis del discurso desde la lingüística cognitiva son armonizables y están orientados a objetivos coincidentes. Esta convergencia se pone de manifiesto en las palabras de Villegas (1993):

Si entendemos por discurso la manifestación del pensar, la comunicación de una intención a otros (Austin, 1962) de una forma directa o inmediata a través de intercambios interactivos — conversación — o de una forma indirecta y mediada a través de textos escritos o de mensajes orales audio-vídeograbados, la cuestión que se plantea es la del acceso a la matriz intencional generadora del discurso, que es la única que garantiza, en último término, su comprensión. Tanto el proceso de producción como de comprensión del discurso implican la referencia a estructuras de conocimiento gramaticales y extra gramaticales que se sitúan en la interfaz entre la actividad cognitiva inteligente y la actividad lingüística, propiamente dicha (Belinchón, 1992). Esta doble dimensión del discurso, cognitiva y lingüística o psicolingüística, donde se combina el procesamiento semántico pragmático con el gramatical, evidencia la duplicidad de niveles en que se realiza. (p. 22)

En la medida en que se analizan espacios de producción de sentido, se ha decidido delimitar el corpus de este artículo con base en la temática de la familia y priorizar los discursos aparecidos en las redes sociales, pues lo que se busca es dilucidar, justamente, el modo en que la red que el artista teje con sus seguidores se modela de una forma particular a través de determinadas conceptualizaciones metafóricas.

El corpus analizado está compuesto de discursos presentes en diversas redes sociales, principalmente, en Facebook, que presentan la metáfora "el público es la familia" para conceptualizar la relación de Abel Pintos con su público. A lo largo del análisis, se irán señalando las características lingüísticas de la comunidad discursiva de los seguidores de Abel Pintos que resulten particulares, recurrentes y relevantes.

Para el análisis del corpus, se utilizarán categorías propias del análisis del discurso desde una perspectiva cognitivo-funcional (Nuyts, 2004). El eje vertebrador de la indagación será la metáfora de la familia abelera, por lo cual el marco teórico central del trabajo está constituido por la teoría de la metáfora conceptual (Lakoff y Johnson, 2001)², una de las principales líneas teóricas de la lingüística cognitiva ${ }^{3}$. Sobre la metáfora conceptual hay ingente material bibliográfico, el cual re-

2 Estos son los iniciadores de la teoría como tal. Algunos desarrollos teóricos recientes sobre el asunto son los de Steen (2008), Gibbs (2011), Steen (2011) y Landau, Robinson y Meier (2013).

3 No es posible realizar una caracterización completa de esta perspectiva de estudio de los fenómenos lingüísticos. Por esta razón, se invita al lector interesado a revisar los trabajos de Cuenca y Hilferty (1999), Croft y Cruse (2008), Geeraerts (2006) e Ibarretxe-Antuñano (2013), entre muchos otros estudios que existen sobre el tema. 
sulta inabarcable por este estudio ${ }^{4}$. Si se desea conocer mejor el desarrollo histórico de la noción de metáfora como concepto y profundizar en su caracterización desde la lingüística cognitiva, se puede consultar el trabajo de Gonzalez (2016).

La teoría de la metáfora conceptual afirma que la metáfora constituye un fenómeno del pensamiento que se refleja en el lenguaje, que está omnipresente en la vida cotidiana y que permite la estructuración de la experiencia. En este sentido, la esencia de la metáfora era entender y experimentar un tipo de cosa en términos de otra. Una metáfora conceptual es la proyección de un dominio fuente sobre un dominio meta. Por ejemplo, en la metáfora "el tiempo es dinero", el dominio fuente es el dinero y el dominio meta es el tiempo. En este caso, la proyección de dominios se produce en expresiones metafóricas como "le entregué mis mejores momentos" y "estás robando mi tiempo".

El enfoque de este estudio se enmarca en la línea de investigaciones que integran el análisis de las metáforas y las metonimias, los discursos en determinados dominios cognitivos y su impacto en la sociedad, los estudios sobre las metáforas acerca del cáncer (Williams Camus, 2009; Semino, Demjén y Demmen, 2016), la metáfora en el discurso político (Britta et al., 2017; Boeynaems et al., 2017), la construcción metafórica de los roles en la educación (Vadeboncoeur y Torres, 2003), la metáfora como uno de los principales recursos para implementar políticas educativas (Cushing, 2019), los patrones metafóricos y metonímicos en las charlas de reconciliación (Cameron, 2007), las metáforas en el campo de la economía (Domínguez, 2015) y la psicología (Tay, 2011).

\section{Resultados}

Se presenta a continuación el resultado del análisis del corpus. Para comenzar, no está de más insistir en que el rasgo más distintivo del fenómeno de masas que se estudia aquí es el hecho de que ciertas figuras públicas (en este caso, un artista) llegan a cohesionar grandes grupos heterogéneos de personas que, a pesar de su diversidad, logran compartir una matriz de sentidos e incluso promoverla.

La siguiente entrada de Facebook y sus comentarios (figura 1) se abordaron como botón de muestra de lo señalado acerca de las particularidades que manifiesta la relación individuo-público-masa entre Abel Pintos y su público.

\footnotetext{
$4 \quad$ Algunos de los trabajos que se pueden destacar son los de Lakoff (1993, 2008), Croft (1993), Gibbs (1996), Geeraerts (2002), Glenberg (2010), Kövecses (2010) y Gonzalez (2013, 2016).
} 


\section{Facu Nichea}

- Admin · January 31 at 5:36 PM

\#FAMILIA!!! Se nos viene el primer aniversario del grupo!

El 13 de febrero se cumple un año desde que se creó este harrrrrrrmoso grupo y voy a hacer un video para celebrarlo. Quienes quieran formar parte del video, sáquense una FOTO con algún cartelito que diga "FAMILIA ABELERA $\bullet "$ "(no se olviden de dibujar el corazón jaja..)

Así las pongo en el video $:$

También dejen en los comentarios fotos de los recitales, de las juntadas entre ustedes, escriban o suban videos contándonos quiénes se han conocido gracias al grupo y todo lo que quieran sumarle, que yo después me encargo de recopilar y armar el video!

Tienen tiempo hasta el 10 de febrero (masssomenos), pero traten de no dejarlo todo para lo último, así puedo ir armándolo de a poquito $:-$

Pd: Gracias Gise de Cristobal por recordarme que estábamos por llegar al primer aniversario!!

$\mathrm{SI}$ a alguien se le ocurre algo más para hacer, comentenlo!

Graciasssssss!!!

Figura 1. Publicación del aniversario Facu Nichea Fuente: Nichea (2019).

Como se puede apreciar, al inicio de esta publicación se encuentra la etiqueta "Familia" enfatizada con las mayúsculas. El concepto más importante del grupo es el de constituir una comunidad con una fuerte unión como la de una familia. Así es clasificado por su administrador el tipo de grupo de Facebook (Familia) y así aparece especificado en la descripción: "FAMILIA!!!!! Muy bienvenidos!!!!!!!!!!! Ojalá que entre todos hagamos de este grupo un lugar de BUENA ENERGÍA!!!! Que cualquiera que necesita algo, tenga siempre a alguien dispuesto a ayudar!!! [sic]" (Familia ablera, 2019).

La idea de que los miembros del grupo conforman una familia es recurrente en las publicaciones y comentarios del grupo. En los comentarios de esta publicación particular que se analiza aquí, aparece manifestada de los siguientes modos:

Claudia Capurro Feliz de formar parte de esta familia Me gusta $\cdot$ Responder $\cdot 1$ año

Andrea Fernandez Siiiiiii hermosa familia.....super orgullosa de pertenecer a esta familia...dónde hay gente INCREÍBLE...

Me gusta $\cdot$ Responder $\cdot 1$ año 
Maribel Vidal Siii....taaann feliz de pertenecer a esta hermosa familia, gracias Facu Nichea por crear este grupo que es lo máximo!!!! ;

Maria Angélica Quevedo Aguante la familia !!!!!!!

Me gusta $\cdot$ Responder $\cdot 1$ año

Mary Muñoz Hermoso grupo..hermosa familia!! ș

Me gusta $\cdot$ Responder $\cdot 1$ año

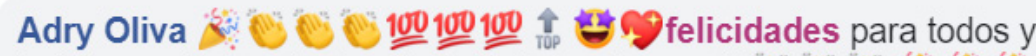
todas grande facu le familia festeja fuerte o qué?

En los comentarios de la publicación, la mayoría de los miembros del grupo celebra la idea del administrador de realizar un video con fotos de todos los miembros y sube sus fotos personales para formar parte de la producción audiovisual. La mayoría de los comentarios emplea la palabra familia. En algunos de los comentarios y fotos, los usuarios hacen autorreferencias como la pregunta "¿o qué?", presente en el comentario de Oliva (2019), que es una frase que comúnmente repite Abel Pintos. También aparecen fragmentos de canciones del artista.

No queremos dejar de advertir la creación de un adjetivo con amplia aceptación y generalización como lo es el de abelera, presente en el nombre del grupo cuya publicación analizamos. En efecto, hasta existen variantes de este término como el que da nombre al grupo de Facebook llamado Abelocas.

Sobre los grupos físicos institucionalizados que mencionábamos arriba, aparece uno de ellos en la que la publicación que se analiza en el siguiente comentario:

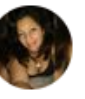

Gise de Cristobal Gracias Facu Nichea x aceptar la propuesta!!! Mañana mandamos la de Alma Abelera en Tunuyán

La metáfora "el público es la familia" de Abel Pintos es repetida por el cantante casi como una consigna, con un lenguaje bastante performativo. Como en toda proyección metafórica, hay aspectos de la fuente que se proyectan en la meta y otros que no. Por otra parte, no todos los seguidores del cantante parecen encarnar la consigna de la misma manera. Por ello, suceden eventos que entran en conflicto con 
la concepción metafórica "el público es la familia" y representan para muchos fanes del artista un choque conceptual. No es de esperar otra cosa de un grupo ideológicamente heterogéneo y amplio como este ni del medio en el que interactúan, las redes sociales, caldo de cultivo de críticas y discusiones. ${ }^{5}$. No hay espacio para analizar in extenso la publicación que se utilizará para ejemplificar este punto. Baste lo dicho y la publicación misma que se presenta en la figura 2.

\section{ovall Ruiz}

24 de noviembre de 2019

FAMILIA si pero a la FAMILIA de verdad... chicas estoy leyendo ya que mucha gente se está quejando de como escribe Romi y de que no saben que es no vidente... y si el echo fuera de que no sabe escribir ella o cualquiera de nosotras por fuera la causa que fuere... estoy viendo demasiada agresión y realmente en una VERDADERA FAMILIA como es la nuestra no me gusta saber de esto y menos ver cómo se le habla al otro... entonces propongo que a la gente que agrede se la saque del grupo si es que no pide las disculpas correspondientes al caso.. me duele la agresión que se está provocando... es mi opinión digan uds que les parece

Figura 2. Opinión fanática para la no agresión Fuente: Ruiz (2019).

Cabe preguntarse si la metáfora de la familia, que el propio artista ha instalado y alimenta en sus seguidores, responde adecuadamente a la situación que se explica con esa proyección o si, más bien, se trata de un sentido de familia como el que se adopta en comunidades religiosas. En efecto, en una familia prototípica hay un número limitado de miembros con una fuerte conexión (de sangre, generalmente), mucho tiempo de convivencia y un alto grado de confianza, mientras que en las masas de seguidores hay numerosas personas, que no se conocen entre sí y solo comparten el gusto por una figura pública y su obra.

La característica principal de las familias que el artista bahiense proyecta en su metáfora es el respeto en el trato con los demás miembros del grupo y las reuniones

Gallardo Camacho (2013) resalta el poder interactivo de las redes y la apertura a las críticas que implican en relación con los presentadores de televisión. Su comentario es útil también para el caso que se analiza aquí: "El presentador 2.0. ha llegado para quedarse pero se enfrenta a un espectador social y participativo. El público es más crítico que antes porque la cadena no es la que elige ahora qué comentario sale a la luz. Todo el mundo es libre de difundir y de opinar sobre los contenidos televisivos. La democracia audiovisual ha llegado con el espectador 2.0. Y es que el espectador social ha cambiado las reglas del juego para los presentadores que se sienten más vigilados y auditados profesionalmente por una masa espontánea que ha devuelto la interactividad a la caja tonta" (p. 18). 
frecuentes. El respeto mutuo es lo que no ha sentido como cumplido la usuaria de Facebook que hace la crítica presentada en la figura 2. Lo que no se proyecta en la metáfora en este caso es el estar disponible para el otro en todo momento y conocer datos de su intimidad, entre otras particularidades.

En la siguiente publicación se aprecian las características positivas de las familias que se proyectan en la metáfora "el público es familia": complicidad, solidaridad y confianza (figura 3).

\section{Belu Nora}

13 de noviembre de 2019

Hola familia quiero contarles, hace un año atrás mi sueño de estar en el concierto del Estadio Obras, por cosas de la vida no se pudo cumplir, y me quedé con el sabor amargo, de haber tenido que vender mi entrada, y con las ganas de viajar a Baires por primera vez. Hoy la vida me dió una nueva oportunidad, de la mano de Carla y quiero resaltar el gesto y complicidad de Romina Anchaval, quien a pesar de su tristeza de tener que vender sus entradas, confió en nosotras y nos envió las entradas, desde Córdoba a Mendoza, aún sin pagarle un peso previo. No puedo explicar la emoción al abrir el paquete y encontrarme con ese cartel que dice: "disfruten mucho", esto habla de complicidad, de solidaridad y confianza. Sólo la familia abelera sabe lo que significa un concierto de Abel para cada un@ de nosotr@s. Y ese cartel lo dice todo! GRATITUD ETERNA ROMI, Y OJALÁ PRONTO NOS ENCONTREMOS EN UN CHOW!

Felicidad absoluta! Sinfónico allá vamos!

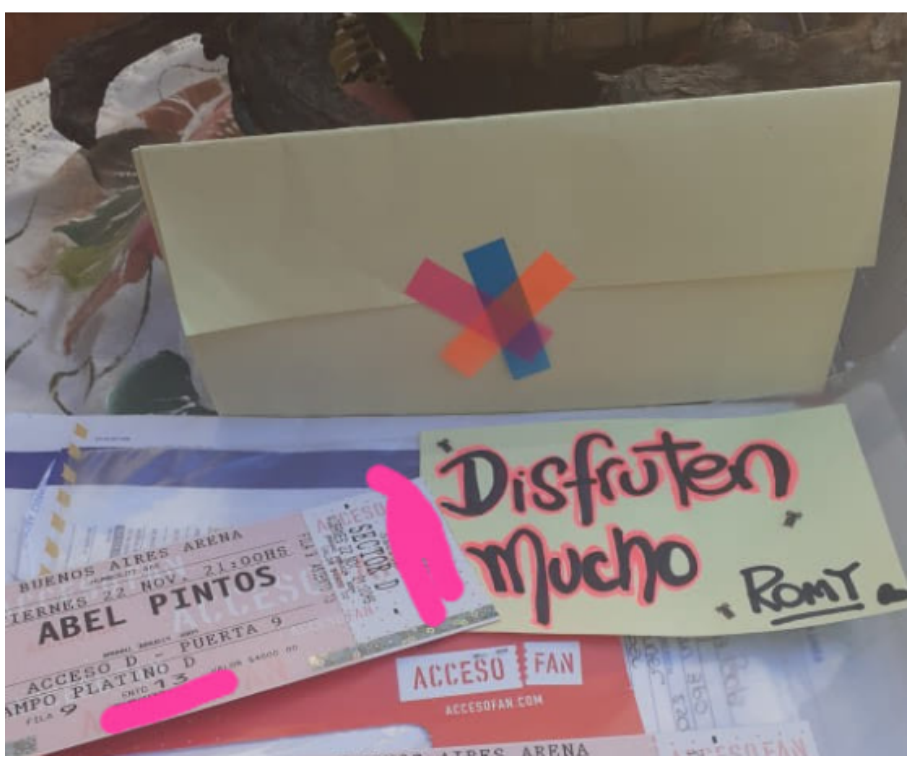

Figura 3. Gratitud de fanática por boletas de concierto Fuente: Nora (2019). 
El panorama de la relación del cantante con sus seguidores no quedaría totalmente elaborado si se ignorara el hecho de que la admiración que despierta su arte es generalizada a todos los niveles de la vida, al punto de que muchos de sus seguidores hablan de "haber sido salvados" por Abel. En la figura 4, se muestra un ejemplo de esto.

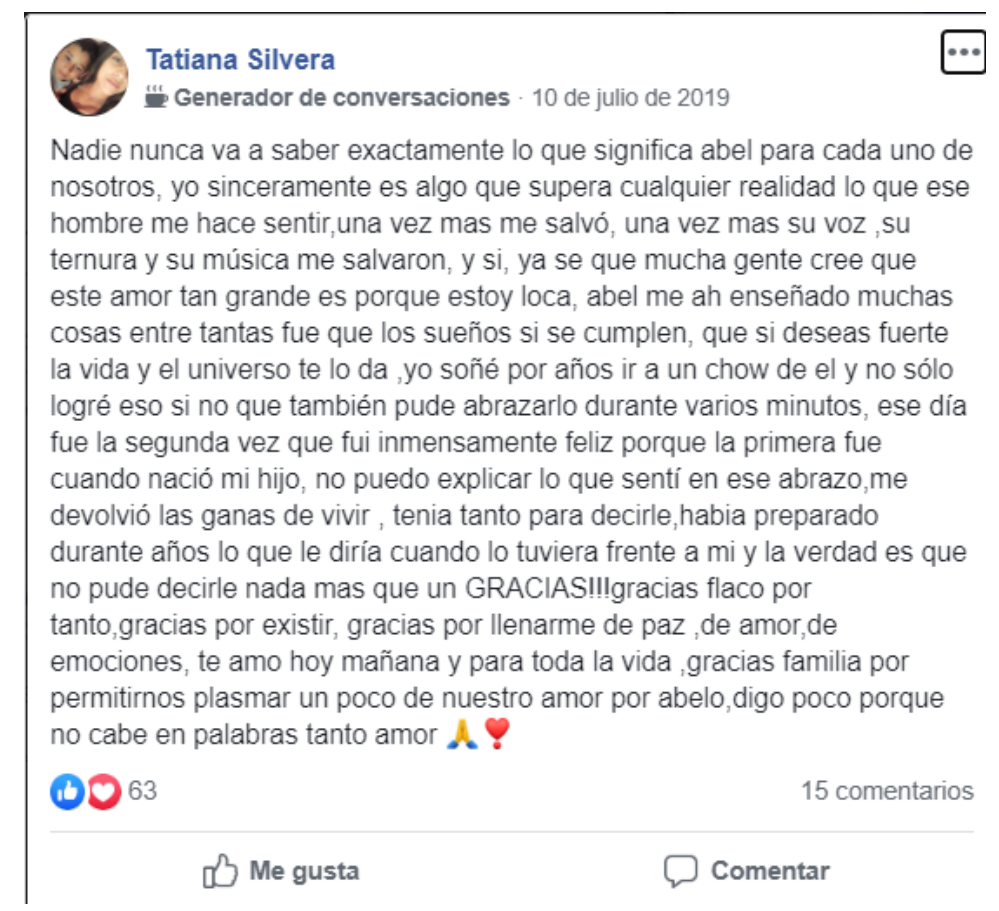

Figura 4. Significado de Abel para una fan Fuente: Silvera (2019).

Otros nombres de grupos de Facebook son los siguientes:

(1) Grupos de Facebook con la idea de familia:

- Familia Abelera Nacional. https://www.facebook.com/groups/1137406376408811/

- Juntas para verte Familia Abelera Bs.As (https://www.facebook.com/groups /210127899728189/.

(2) Grupos que contienen el adjetivo abelero en su nombre.

- Sentimiento Abelero. https://www.facebook.com/groups/401837490636182/.

- Abeleras/os Trelewenses. https://www.facebook.com/AbelerasT/.

- Abeler@s AP. https://www.facebook.com/groups/1497249470562756/ 
(3) Grupos con referencias a canciones del artista:

- El bosque de Abel (mariposas, pájaros cantores, adivinos y brujas). https://www.facebook. com/groups/210084476434154/

- Flor Azul AP. https:/www.facebook.com/groups/FLORAZULAP/

- Motivos. https://www.facebook.com/groups/729290330802823/

- Pájaro cantor. https://www.facebook.com/groups/345130282632613/

- Que fortuna encontrarte Abel Pintos https://www.facebook.com/ groups/693205110732032/

- Abel Pintos-Complises =). https://www.facebook.com/groups/610081305694555/

(4) Grupos con referencias internas (nombres de canciones, comentarios de Abel Pintos) que hacen alusión al amor erótico que las seguidoras sienten por el cantante:

- Ternuritas Patagonicas (Pcia.Rio Negro). https://www.facebook.com/ groups/222769904946593/.

- Te Amo Abel Federico Pintos. https://www.facebook.com/groups/1733354793581955/

- Ternuritas \#PELD. https://www.facebook.com/groups/838591819535178/

- Mariposas de Abel Mendoza. https://www.facebook.com/groups/768582493312856/

- Mariposas Uruguayas. https://www.facebook.com/groups/1983926648567334/

(5) Otros grupos que hacen alusión al amor erótico que las seguidoras sienten por el cantante:

- Las nenas de Abel Pintos AP. https://www.facebook.com/groups/1844867019139753/

Sobre esta sigla, AP, es oportuno señalar que muchos usuarios de Facebook la colocan luego de su nombre para marcar su fanatismo/amor por el cantante como en los siguientes ejemplos hallados en Facebook: Ani Acuña AP, K-ro AP y Tamiïh Casm SJ AP, Derrapador@s de AP y las derraperas de abel.

\section{Discusión y conclusiones}

El análisis de la metáfora "el público es la familia" que se ha llevado a cabo en este artículo, permite apreciar que la conceptualización metafórica promocionada por el cantante Abel Pintos para hacer referencia a la relación con sus seguidores ha sido adoptada por gran parte de su público. Dicho público exhibe muchas de las características principales de los movimientos de masas, entre ellas, un nivel de cohesión alto, la organización para fines comunes y el respaldo y la admiración por el cantante. Esto se pone de manifiesto a través de referencias internas, símbolos en común y uso de productos con la marca del artista. 
El análisis discursivo de algunas publicaciones y comentarios de Facebook permitió apreciar que, efectivamente, el cantante Abel Pintos conceptualiza a su público como una familia y sus seguidores se consideran parte de dicha familia. Así lo afirman constantemente en las redes sociales y se esfuerzan por comportarse de modo familiar entre sí. Este "modo familiar" se observa en el trato respetuoso que conservan y en las actividades grupales que organizan continuamente en las redes sociales y en persona. De hecho, cuando sucede un hecho que rompe con la lógica de la metáfora "el público es la familia" surgen conflictos. La idea de familia también se hace presente en los discursos que afirman que el cantautor bahiense ha "salvado" las vidas de quienes los escriben porque les ha aportado elementos para resignificar diversos eventos difíciles de procesar o traumáticos.

Hay muchas otras metáforas de la relación del público de Abel Pintos con el artista, pero no son analizadas en este trabajo por falta de espacio. Para futuros estudios se pueden analizar las conceptualizaciones previas del cantautor (peregrinos, cómplices) y las que actualmente circulan entre los seguidores de Abel, entre ellas, la de mariposas, ternuritas y flores. Por supuesto, al analizar cada metáfora se pueden apreciar los diversos sistemas conceptuales a ellas asociados.

El análisis realizado permite apreciar que el estudio de las metáforas conceptuales en ámbitos muy específicos y no abordados comúnmente desde la lingüística (en este caso, el de la relación de un cantante con su público) permite explicar algunos aspectos de dichos ámbitos y, en consecuencia, los comportamientos de los grupos sociales involucrados. Además, aporta a las diversas disciplinas lingüísticas elementos para comprobar el funcionamiento de las teorías lingüísticas y describir diversas configuraciones dentro de las diferentes comunidades discursivas.

\section{Referencias}

Ajmat, S. (2017, 12 de noviembre). En la ruta con Abel Pintos, el ídolo que insiste en ser libre. La Nación. https://www.lanacion.com.ar/2080883-en-la-ruta-con-abel-pintos-el-idolo-popular-queinsiste-en-ser-libre

Britta, C., Brugman, C.; Burgers, G. y Steen, J. (2017). Recategorizing Political Frames: a Systematic Review of Metaphorical Framing in Experiments on Political Communication. Annals of the International Communication Association, 41(2), 181-197. 10.1080/23808985.2017.1312481

Boeynaems, A., Burgers, C., Konijn, E y Steen, G. (2017). The Effects of Metaphorical Framing on Political Persuasion: A Systematic Literature Review. Metaphor and Symbol, 32(2), 118134. 10.1080/10926488.2017.1297623

Cameron, L. (2007). Patterns of Metaphor Use in Reconciliation Talk. Discourse \& Society, 18(2), 197-222. https://doi.org/10.1177/0957926507073376

Capurro, C. [@ClaudiaCapurro] (2019). Feliz de formar parte de esta familia. |Comentario a publicación de Facebook]. Facebook. Recuperado el 30 de mayo de 2020. https://www. 
facebook.com/groups/219054015335939/search/?query=Facu\%20Nichea\%20genial...\%20 este\%20grupo\%20es\%20hermoso\%20la\%20verdad\%20...\%20desde\%20que.me\%20 un\%C3\%AD\%20es\%20una\%20gran\%20familia\&epa=SEARCH_BOX

Croft, W. (1993). The Role of Domains in the Interpretation of Metaphors. Cognitive Linguistics, $4(4), 335-371$.

Croft, W. y Cruse, D. (2008). Lingüística cognitiva [trad. A. Benítez Burraco]. Akal.

Cuenca, M. y Hilferty, J. (1999). Introducción a la Lingüística Cognitiva. Ariel.

Cushing, I. (2019). The Policy and Policing of Language in Schools. Language in Society, 1-26.

De Cristobal, G. [@GisedeCristobal] (2019, 31 de enero). Gracias Facu Nichea x aceptar la propuesta!!! Mañana mandamos la [foto] de Alma Abelera en Tunuyán 8 . [Imagen adjunta] [Comentario a publicación de Facebook]. Recuperado el 30 de mayo de 2020. https://www. facebook.com/groups/219054015335939/search/?query=se\%20nos\%20viene\%20el\%20 primer\%20aniversarioEepa $=$ SEARCH_BOX

Domínguez, M. (2015). The Metaphorical Species: Evolution, Adaptation and Speciation of Metaphors. Discourse Studies, 17(4), 433-448. https://doi.org/10.1177/1461445615578963

Familia abelera [Grupo de Facebook]. (2019, 31 de enero). Descripción del grupo [Actualización de estado]. Facebook. Recuperado el 30 de marzo de 2019. https://www.facebook.com/ groups/219054015335939/

Familia abelera [Grupo de Facebook]. (2019, 15 de febrero). Descripción del grupo [Actualización de estado]. Facebook. Recuperado el 30 de marzo de 2019. https://www.facebook.com/ groups/219054015335939/

Fernandez, A. [@ClaudiaCapurro] (2019). Siiiiii hermosa familia. [Comentario a publicación de Facebook]. Recuperado el 30 de mayo de 2020. https://www.facebook.com/ groups/219054015335939/search/?query=Facu\%20Nichea\%20genial...\%20este\%20grupo\%20 es\%20hermoso\%20la\%20verdad\%20....\%20desde\%20que.me\%20un\%C3\%AD\%20es\%20 una\%20gran\%20familiaEepa=SEARCH_BOX

Gallardo Camacho, J. (2013). El presentador de televisión y su relación con las redes sociales en España. Caso "Espejo Público" (Antena 3). En B. Lloves Sobrado y F. Segado Boj (coords.), I Congreso Internacional de Comunicación y Sociedad Digital. Logroño.

Geeraerts, D. (2002). The Interaction of Metaphor and Metonymy in Composite Expressions. En R. Dirven; R. Pörings (ed.), Metaphor and metonymy in comparison and contrast (pp. 435-465). Mouton de Gruyter.

Geeraerts, D. (ed.) (2006). Cognitive Linguistics: Basic Readings. Mouton de Gruyter.

Gibbs, R. (1996). What's cognitive about cognitive linguistics? En E. Casad (ed.), Linguistics in the Redwoods: The Expansion of a New Paradigm in Linguistics (pp. 27-53). Mouton de Gruyter.

Gibbs, R. (2011). Evaluating Conceptual Metaphor Theory. Discourse Processes, 48(8), 529562. $10.1080 / 0163853 X .2011 .606103$

Glenberg, A. (2010). Embodiment as a Unifying Perspective for Psychology. Cogn Sci, 1(4), 586-596. 
Gómez, M. (2018, 26 de octubre). Sueño cumplido: Abel Pintos festejó fuerte con su "familia" en el templo del rock. Clarín. https://www.clarin.com/espectaculos/musica/abel-pintos-festejo-fuerte-familiatemplo-rock_0_K2DWvZOxd.html

Gonzalez, D. (2013). "La metáfora desde la perspectiva cognitivista de Lakoff y Johnson". En: M. Rodríguez (ed.), La metáfora, Mendoza: SSECC-CEFIC, 109-129.

Gonzalez, D. (2016). La Lingüística Cognitiva y su teorización sobre la metáfora conceptual: Principales visiones históricas sobre el fenómeno y caracterización desde los principios cognitivo-funcionales. España: Editorial Académica Española.

Ibarretxe-Antuñano, I. (2013). La lingüística cognitiva y su lugar en la historia de la lingüística. Resla, 26, 245-266.

Kövecses, Z. (2010). Metaphor: A Practical Introduction (2 $2^{\mathrm{a}}$ ed.). Oxford University Press.

Lakoff G. y Johnson M. (2001). Metáforas de la vida cotidiana [trad. J. Millán y S. Narotzky] (5ª ed). Cátedra.

Lakoff, G. (1993). The Contemporary Theory of Metaphor. En A. Ortony (ed.), Metaphor and Thought, $2^{a}$ ed. (pp. 202-251). Cambridge University Press.

Lakoff, G. (2008). The neural theory of metaphor. En R. Gibbs (ed.), The Cambridge Handbook of Metaphor and Thought (pp. 17-38). Cambridge University Press.

Landau, M., Robinson, M. y Meier, B. (eds.). (2013). The Power of Metaphor: Examining its Influence on Social Life. American Psychological Association.

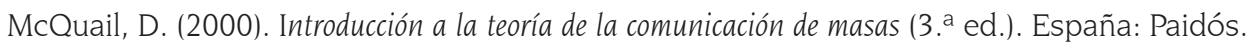

Muñoz, M. [@MaryMuñoz] (2019). Hermoso grupo... hermosa familia!!. [Comentario a publicación de Facebook]. Recuperado el 30 de mayo de 2020. https://www.facebook.com/ groups/219054015335939/search/?query=Facu\%20Nichea\%20genial...\%20este\%20grupo\%20 es\%20hermoso\%20la\%20verdad\%20...\%20desde\%20que.me\%20un\%C3\%AD\%20es\%20 una\%20gran\%20familiaEepa=SEARCH_BOX

Nichea, F. [@FacuNichea] (2019, 31 de enero): \#FAMILIA!!! Se nos viene el primer aniversario del grupo [Publicación de Facebook en grupo Familia abelera]. Recuperado el 30 de mayo de 2020 : https:// www.facebook.com/groups/2 19054015335939/search/?query=se\%20nos\%20viene\%20el\%20 primer\%20aniversario\&epa $=$ SEARCH_BOX

Nora, B. [@BeluNora] (2019, 13 de noviembre). Hola familia quiero contarles, hace un año atrás mi sueño de estar en el concierto del Estadio Obras (...)". [Publicación de Facebook en el grupo Familia abelera]. Recuperado el 30 de mayo de 2020. https:/www.facebook.com/groups/219054015335939/ permalink/561709147737089/

Nuyts, J. (2004). The Cognitive- Pragmatic Approach. Intercultural Pragmatics, 1(1), 135-149.

Quevedo, M. [@MariaAngélicaQuevedo] (2019). Aguante la familia !!!!!!. [Comentario a publicación de Facebook]. Recuperado el 30 de mayo de 2020. https://www.facebook.com/ groups/2 19054015335939/search/?query=Facu\%20Nichea\%20genial...\%20este\%20grupo\%20 es\%20hermoso\%20la\%20verdad\%20....\%20desde\%20que.me\%20un\%C3\%AD\%20es\%20 una\%20gran\%20familiaEepa=SEARCH_BOX 
Ruiz, V. [@VallRuiz] (2019, 24 de noviembre). FAMILIA si pero a la FAMILIA de verdad. [Publicación de Facebook en el grupo Familia abelera]. Recuperado el 30 de mayo de 2020. https://www. facebook.com/groups/219054015335939/permalink/570679133506757/

Semino, E., Demjén, Z. y Demmen, J. (2018). An Integrated Approach to Metaphor and Framing in Cognition, Discourse, and Practice, with an Application to Metaphors for Cancer. Applied Linguistics, 39(5), 625-645. https://doi.org/10.1093/applin/amw028

Silvera, T. @TatianaSolvera (2019,10 de julio). Nadie nunca va a saber exactamente lo que significa abel para cada uno de nosotros. [Publicación de Facebook en el grupo Familia abelera]. Recuperado el 30 de mayo de 2020. https://www.facebook.com/groups/219054015335939/ permalink/482625638978774/

Steen, G. (2011) Metaphor, Language, and Discourse Processes. Discourse Processes, 48(89), 585591. 10.1080/0163853X.2011.606424

Steen, G. (2008). The Paradox of Metaphor: Why we need a Three-Dimensional Model of Metaphor. Metaphor and Symbol, 23(4), 213-41.

Tay, D. (2011). Therapy is a Journey as a Discourse Metaphor. Discourse Studies, 13(1), 47-68. https:// doi.org/10.1177/1461445610387736

Vadeboncoeur, J. y Torres, M. (2003). Constructing and Reconstructing Teaching Roles: A Focus on Generative Metaphors and Dichotomies. Discourse: Studies in the Cultural Politics of Education, 24(1), 87-103. 10.1080/01596300303034

Vidal, M. [@MaribelVidal] (2019).Siii....taaann feliz de pertenecer a esta hermosa familia. [Comentario a publicación de Facebook]. Recuperado el 30 de mayo de 2020. https://www.facebook.com/ groups/219054015335939/search/?query=Facu\%20Nichea\%20genial...\%20este\%20grupo\%20 es\%20hermoso\%20la\%20verdad\%20...\%20desde\%20que.me\%20un\%C3\%AD\%20es\%20 una\%20gran\%20familiaEepa $=$ SEARCH_BOX

Villegas, M. (1993). Las disciplinas del discurso: hermenéutica, semiótica y análisis textual. Anuario de psicología, 59, 19-60.

Williams Camus, J. (2009). Metaphors of Cancer in Scientific Popularization Articles in the British Press. Discourse Studies, 11(4), 465-495. https://doi.org/10.1177/1461445609105220 